\title{
ZONEAMENTO DE RISCO DE INCÊNDIOS FLORESTAIS PARA O ESTADO DO PARANÁ
}

\author{
Danielle dos Santos de Oliveira ${ }^{1}$ \\ Antonio Carlos Batista ${ }^{2}$ \\ Ronaldo Viana Soares ${ }^{3}$ \\ Leocádio Grodzki ${ }^{4}$ \\ Jackson Vosgerau ${ }^{5}$
}

\begin{abstract}
RESUMO
O objetivo deste trabalho foi obter o Zoneamento de Risco de Incêndio Florestal para o estado do Paraná (ZRIFPR), considerando o efeito integrado da presença humana, cobertura vegetal, condições meteorológicas e características topográficas. Para esta análise utilizou-se um Sistema de Informações Geográficas. Foram preparados mapas de risco preliminares para cada uma das variáveis em estudo. Estes mapas foram sobrepostos, e o resultado deste cruzamento de informações resultou no ZRIF-PR. De acordo com o ZRIF-PR, 51,87\% da área foi classificada como risco moderado e $30,16 \%$ como risco alto. Para a validação do ZRIF-PR, o mesmo foi comparado com o mapa de focos de calor e o mapa das ocorrências de incêndio registradas entre 1991 e 2001. O modelo de integração proposto é o mais indicado para gerar o ZRIF-PR porque emprega maior número de variáveis e foi elaborado a partir de condições ambientais similares às do Paraná.

Palavras chave: presença humana; condições meteorológicas; topografia; SIG; proteção florestal
\end{abstract}

\begin{abstract}
The objective of this research was develop a forest fire risk map through the integrated analysis of human presence, vegetation cover, meteorological variables, fuel moisture, elevation, slope gradient and aspect. For this analysis the Geographical Information System (GIS) was used. The forest risk map (ZRIF-PR) was the result from the superposition of the thematic maps. The fire risk map obtained showed that $51,8 \%$ of the area was under moderate risk and $30,16 \%$ under high risk.
\end{abstract}

Key words: human presence; meteorological variables; topography; GIS; forest protection

\section{INTRODUÇÃO}

A ação do fogo sobre a floresta atinge não só a cobertura vegetal, mas também as características do solo, a fauna e a atmosfera, podendo ser altamente destrutiva quando se trata de incêndios florestais, como o que ocorreu no estado do Paraná em 1963. Neste incêndio, foram atingidos 2 milhões de hectares (aproximadamente $10 \%$ da superfície do estado) de florestas nativas, reflorestamentos e outros tipos de vegetação. Além disso, mais de 5.000 casas foram destruídas e 110 pessoas foram mortas pelo fogo (Batista; Oliveira; Soares, 2002).

Apesar do aperfeiçoamento das técnicas de prevenção e combate desenvolvidas desde então, incêndios florestais continuam a ocorrer no Estado. Estes incêndios têm sido observados em anos de inverno mais secos, que se sucedem a cada três a cinco anos, de acordo com observações meteorológicas disponíveis. A freqüência com que o Estado é atingido por incêndios justifica a elaboração do Zoneamento de Risco de Incêndio Florestal do Paraná (ZRIF-PR).

Assim, o objetivo principal deste trabalho foi apresentar a metodologia para elaboração do ZRIF-PR, usando um Sistema de Informações Geográficas (SIG). Este trabalho foi desenvolvido pelo Laboratório de Incêndios Florestais da UFPR, em parceria com o IAP, IAPAR e SEMA, com financiamento da Fundação Araucária.

O ZRIF-PR é um mapa que apresenta o terreno subdividido em áreas delimitadas em função do potencial de ocorrência e propagação de incêndios. Estas áreas são

\footnotetext{
${ }^{1}$ danielleso@pop.com.br - Eng.Cartógrafa, Msc., IBGE, R. Martinho di Haro, 205 - Apto. 101 A - Ingleses - 88058-540 - Florianópolis/SC

2 batista@floresta.ufpr.br - Prof. Dr. UFPR, R. Lothário Meissner, 3400 - Jd. Botânico - 80210-170 - Curitiba/PR.

3 rvsoares@floresta.ufpr.br - Prof. Phd. UFPR, R. Lothário Meissner, 3400 - Jd. Botânico - 80210-170 - Curitiba/PR.

${ }^{4}$ Eng. Agrônomo, Dr., IAPAR, Curitiba/PR.

${ }^{5}$ Eng. Florestal, IAP, Curitiba/PR.
} 
identificadas através da análise de diferentes características ambientais, que associadas se traduzem em um mesmo potencial de risco. Os fatores do ambiente mais relacionados com os incêndios florestais são o material combustível, as condições climáticas, a topografia e o tipo de cobertura vegetal (Brown; Davis, 1973).

\section{MATERIAIS E MÉTODOS}

Para obter o ZRIF-PR foram utilizados: o mapa digital de municípios do Estado (SEMA), dados de densidade demográfica por município (SEMA), mapa digital com as unidades de conservação estaduais (SEMA), mapa digital de uso do solo (SEMA/IAPAR), mapa digital da malha viária estadual (SEMA), mapas climáticos (IAPAR), dados altimétricos do Estado (IAPAR), registros de ocorrência de incêndio do período de 1991 a 2001 (Corpo de Bombeiros) e banco de dados com a distribuição dos focos de calor observados em imagens de satélite durante os anos de 2000 e 2001 (INPE). Estes dados foram processados com o auxílio dos seguintes programas: ArcView GIS, com a extensão Spatial Analyst e Microsoft Excel.

A metodologia consistiu na elaboração de mapas de risco preliminares para cada variável em estudo: densidade demográfica, sistema viário, cobertura vegetal, condições climáticas, umidade do material combustível, declividade do terreno, orientação das encostas e altitude.
Em seguida, estes mapas foram integrados com o auxílio de um modelo de ponderação que indicou a importância de cada variável para a ocorrência e propagação dos incêndios no Estado. O ZRIF-PR resultante foi comparado aos mapas com a distribuição espacial dos incêndios registrados pelo Corpo de Bombeiros e dos focos de calor registrados pelo INPE. Esta comparação foi feita para avaliar a confiabilidade do ZRIF-PR obtido.

Os mapas de densidade demográfica e sistema viário indicam a probabilidade de ignição. $\mathrm{O}$ estudo da cobertura vegetal representa a vulnerabilidade ao fogo de acordo com a atividade ocorrente em cada região do Estado. Os mapas de condições climáticas, umidade do material combustível, orientação das encostas e altitude indicam indiretamente o conteúdo de umidade do material combustível influenciando a taxa de propagação dos incêndios. A declividade do terreno está associada à velocidade e direção de propagação.

A análise do risco de incêndio em um ambiente SIG requer que todas as variáveis estejam representadas por meio de mapas temáticos subdivididos em unidades de análise, que neste têm dimensões de 873 x $873 \mathrm{~m}$. Cada mapa de risco preliminar foi obtido a partir da atribuição de um coeficiente de risco para cada unidade de análise, de acordo com uma tabela específica para cada variável ambiental. As tabelas de risco utilizadas para classificação dos mapas preliminares são apresentadas a seguir. Os coeficientes de risco apresentados nestas tabelas traduzem o nível de risco de cada classe de dados e são a base para as operações algébricas posteriores.

Tabela 1: Classificação da densidade demográfica, adaptada de Guillermo Julio (1992)

\begin{tabular}{ccc}
\hline DENSIDADE DEMOGRÁFICA $(\mathrm{hab} / \mathrm{km} 2)$ & RISCO & COEFICIENTES \\
\hline Até 40 & Baixo & 1 \\
$40-60$ & Moderado & 2 \\
$60-80$ & Alto & 3 \\
$80-100$ & Muito alto & 4 \\
Acima de $100 \mathrm{~m}$ & Extremo & 5 \\
\hline
\end{tabular}

Tabela 2: Classificação da distribuição do sistema viário, adaptada de Salas e Chuvieco (1994)

\begin{tabular}{lcc}
\multicolumn{1}{c}{ CLASSES } & RISCO & COEFICIENTES \\
\hline Sob influência & Sim & 1 \\
Sem influência & Não & 0 \\
\hline
\end{tabular}


Tabela 3: Classificação da cobertura vegetal

\begin{tabular}{ccc}
\hline COBERTURA VEGETAL & RISCO & COEFICIENTES \\
\hline \multirow{3}{*}{ Agricultura } & Moderado & 2 \\
& Alto & 3 \\
& Muito Alto & 4 \\
\hline \multirow{2}{*}{ Floresta } & Muito Alto & 4 \\
& Extremo & 5 \\
\hline \multirow{2}{*}{ Pastagem } & Alto & 3 \\
& Muito Alto & 4 \\
\hline Reflorestamento & Extemo & 5 \\
\hline Vegetação Secundária & Extremo & 5 \\
\hline Outros tipos de vegetação & Baixo & 1 \\
\hline
\end{tabular}

Tabela 4: Classificação das condições climáticas

\begin{tabular}{lcc}
\hline ÍNDICE DE RISCO CALCULADO & RISCO & COEFICIENTES \\
\hline$>71$ & Baixo & 1 \\
36 a 71 & Moderado & 2 \\
0 a 35 & Alto & 3 \\
-1 a -36 & Muito alto & 4 \\
-73 a -35 & Extremo & 5 \\
\hline
\end{tabular}

Tabela 5: Classificação da umidade do material combustível

\begin{tabular}{ccc}
\hline UMIDADE DO MATERIAL COMBUSTÍVEL $(\%)$ & RISCO & COEFICIENTES \\
\hline$>15,44$ & Baixo & 1 \\
14,55 a 15,43 & Moderado & 2 \\
13,66 a 14,54 & Alto & 3 \\
12,77 a 13,65 & Muito alto & 4 \\
$<12,77$ & Extremo & 5 \\
\hline
\end{tabular}

Tabela 6: Classificação da declividade do terreno, adaptada de Soares (1985)

\begin{tabular}{ccc}
\hline INCLINAÇÃO $(\%)$ & RISCO & COEFICIENTES \\
\hline Até 15 & Baixo & 1 \\
$16-25$ & Moderado & 2 \\
$26-35$ & Alto & 3 \\
$36-45$ & Muito alto & 4 \\
$\geq 46$ & Extremo & 5 \\
\hline
\end{tabular}

Tabela 7: Classificação da orientação das encostas, adaptada de Ferraz e Vetorrazzi (1998)

\begin{tabular}{lccc}
\hline ÂNGULO DE ORIENTAÇÃO & EXPOSIÇÃO & RISCO & COEFICIENTES \\
\hline $112,5^{\circ}$ a $247,5^{\circ}$ & SE / S / SW & Baixo & 1 \\
$67,5^{\circ}$ a $112,5^{\circ}$ & E & Moderado & 2 \\
$22,5^{\circ}$ a $67,5^{\circ}$ & $\mathrm{NE}$ & Alto & 3 \\
$247,5^{\circ}$ a $337,5^{\circ}$ & $\mathrm{NW} / \mathrm{W}$ & Muito alto & 4 \\
$337,5^{\circ}$ a $22,5^{\circ}$ & $\mathrm{N}$ & Extremo & 5 \\
\hline
\end{tabular}


Tabela 8: Classificação da altimetria, adaptada de salas e Chuvieco (1994)

\begin{tabular}{ccc}
\hline CLASSE ALTIMÉTRICA & RISCO & COEFICIENTES \\
\hline Até $600 \mathrm{~m}$ & Extremo & -1 \\
$600-900 \mathrm{~m}$ & Muito Alto & -2 \\
$900-1200 \mathrm{~m}$ & Alto & -3 \\
$1200-1500 \mathrm{~m}$ & Moderado & -4 \\
Acima de $1500 \mathrm{~m}$ & Baixo & -5 \\
\hline
\end{tabular}

A descrição completa dos procedimentos para a elaboração destes mapas preliminares pode ser observada em FUPEF (2002).

O mapa de risco final foi oriundo da álgebra entre os mapas de risco preliminares (Chuvieco; Congalton, 1989; Salas; Chuvieco, 1994, Sousa et al. 2000; Gouma; Chronopoulou-Sereli, 1998; Ferraz; Vettorazzi, 1998; Hendrix; Coen, 2001). Estes mapas foram integrados por meio de uma soma ponderada adaptada de Oliveira et al. (2002).

$$
\begin{gathered}
\mathrm{RIF}= \\
{[0,52 * \mathrm{DD}+0,52 * \mathrm{SV}]+[(0,29 * \mathrm{D} \%+0,11 * \mathrm{OE}} \\
-0,1 * \mathrm{AL})+ \\
(0,305 * \mathrm{CC}+0,305 * \mathrm{UW})+0,13 * \mathrm{CV}
\end{gathered}
$$

Onde:

RIF - risco de incêndio florestal calculado em cada unidade de análise

DD - variável densidade demográfica

SV - variável distribuição do sistema viário

$\mathrm{D} \%$ - variável declividade do terreno

$\mathrm{OE}$ - variável orientação das encostas

$\mathrm{AL}$ - variável altimetria

CC - variável condições climáticas

UW - variável umidade do material combustível

$\mathrm{CV}$ - variável cobertura vegetal

A aplicação desta somatória ponderada possibilitou a integração dos mapas de risco preliminares, resultando no ZRIF-PR. Este mapa foi comparado aos mapas com a distribuição espacial dos focos de calor e das ocorrências de incêndio, através de uma operação de subtração. Desta forma, quanto maior a freqüência de valores iguais a 0 , maior é a concordância entre as classes de risco atribuídos em ambos os mapas.

\section{RESULTADOS E DISCUSSÃO}

$O$ resultado da integração dos mapas de risco preliminares pode ser observado na Figura 01. O ZRIF-PR indica que $9,96 \%$ da área foi classificada como risco baixo, 51,87\%, como risco moderado, $30,16 \%$ como risco alto, $6,91 \%$ como risco muito alto e 1,09 como risco extremo.

A partir da comparação entre o mapa de risco de acordo com os focos de calor, observouse que $52 \%$ das diferenças apresentadas ficou entre 0 e 1 . A comparação entre o ZRIF-PR e o mapa de risco segundo a distribuição das ocorrências registradas no estado, mostrou que $65 \%$ das diferenças entre 0 e 1 . Estes valores indicam que mais de $50 \%$ da área do estado foi distribuída em classes de risco semelhantes no ZRIF-PR e nos mapas de validação.

O ZRIF-PR apresenta uma macro visão do problema dos incêndios florestais no Paraná. Por isto permanece a necessidade de um detalhamento posterior de áreas consideradas relevante para melhorar a análise do risco no Estado. 


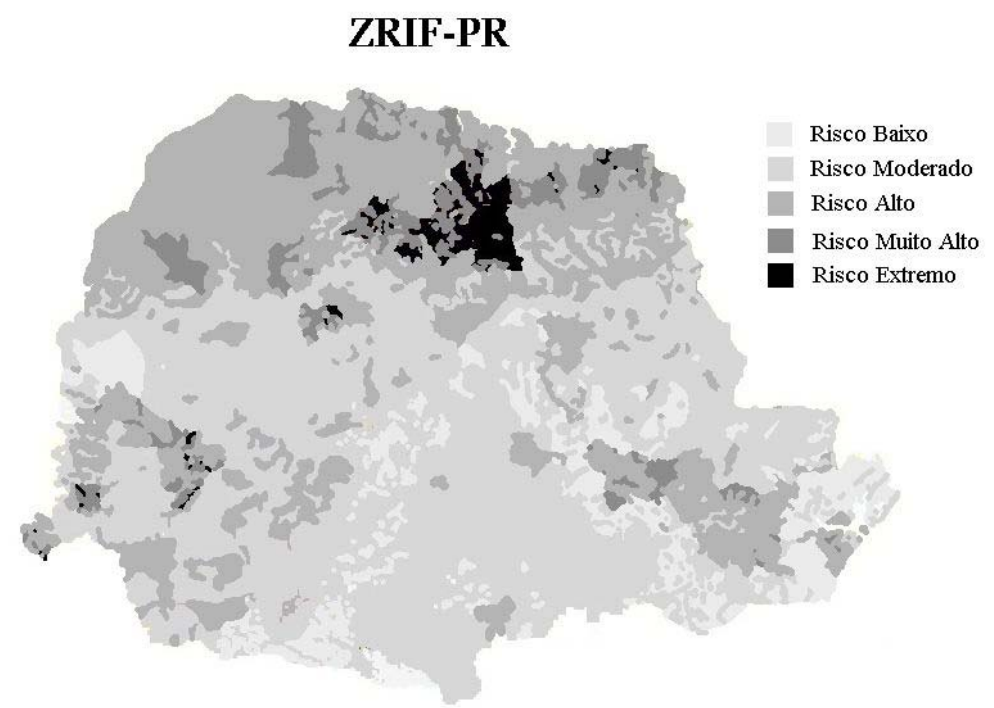

Figura 1: Zoneamento de risco de incêndios floretais para o Estado do Paraná.

\section{CONCLUSÕES}

Considerando as comparações efetuadas entre o mapa obtido e os mapas de focos de calor e o mapa de ocorrências de incêndio, pode-se afirmar que a metodologia apresentada neste trabalho mostrou-se eficiente para representar o risco de incêndio florestal no Estado do Paraná.

O ZRIF-PR deve ser atualizado periodicamente, pois as condições ambientais estão em constante mudança o que pode alterar as condições de risco com o passar do tempo.

\section{REFERÊNCIAS}

BROWN, A. A.; DAVIS, K. P. Forest fire: control and use. 2. ed. New York: McGraw Hill Book, 1973.

CHUVIECO, E.; CONGALTON, R. G. Application of Remote Sensing and Geographic Information Systems to Forest Fire Hazard Mapping. Remote Sensing of Environment, New York, v. 29, p. 147-159, 1989.

FERRAZ, S. F. B.; VETTORAZZI, C. A. Mapeamento de risco de incêndios florestais por meio de sistema de informações geográficas (SIG). Scientia Forestalis, Piracicaba, v. 53, p. 39-48, jun. 1998.

BATISTA, A. C.; OLIVEIRA, D. S.; SOARES, R. V., Zoneamento de Risco de
Incêndios Florestais para o estado do Paraná. Curitiba: FUPEF, 2002.

GOUMA, V.; CHRONOPOULOU-SERELI, A. Wildland fire danger zoning - a methodology. International Journal of Wildland Fire, Colorado, v. 8, n. 1, p. 37-43, 1998.

GUILlERMO JULIO, A. Manual $\mathbf{n}^{\circ} \mathbf{1 0}-$ Método de determinación de prioridades de protección. Universidad de Chile, Facultad de Ciencias Agrarias y Forestales. Departamento de Manejo de Recursos Forestales. Santiago, 27 p., 1992.

HENDRIX, C.; COEN, J. New techniques seek to extinguish wildfire risks. Imaging Notes, Colorado, p. 22-23, 2001.

OLIVEIRA, D. S. Zoneamento de risco de incêndios em povoamentos florestais no norte de Santa Catarina. Universidade Federal do Paraná, Curso de Pós-Graduação em Ciências Florestais, Curitiba. (Dissertação de mestrado). 113 p., 2002.

SALAS, J.; CHUVIECO, E. Geographic information systems for wildland fire risk mapping. Wildfire, Washington, v. 3, n.2, p. 713, jun. 1994.

SOARES, R. V. Incêndios florestais: controle e uso do fogo. Curitiba: FUPEF, 1985.

SOUSA, C. et al. Relatório do Projeto de Cartografia de Risco de Incêndio Florestal CRIF $2^{\mathbf{a}}$ Fase. Disponível em: < www.terravista.pt > Acesso em: 17 abr. 2000. 